\title{
14-3-3 $\lambda$ protein interacts with ADF1 to regulate actin cytoskeleton dynamics in Arabidopsis
}

\author{
ZHAO ShuangShuang ${ }^{1,2}$, ZHAO YanXiu ${ }^{1 *} \&$ GUO Yan $^{2 *}$ \\ ${ }^{1}$ Key Laboratory of Plant Stress, Life Science College, Shandong Normal University, Jinan 250014, China, \\ ${ }^{2}$ State Key Laboratory of Plant Physiology and Biochemistry, College of Biological Sciences, China Agricultural University, Beijing 100193, \\ China
}

Received March 3, 2015; accepted March 28, 2015; published online August 6, 2015

\begin{abstract}
Actin cytoskeleton dynamics is critical for variety of cellular events including cell elongation, division and morphogenesis, and is tightly regulated by numerous groups of actin binding proteins. However it is not well understood how these actin binding proteins are modulated in a physiological condition by their interaction proteins. In this study, we describe that Arabidopsis 14-3-3 $\lambda$ protein interacted with actin depolymerizing factor 1 (ADF1) in plant to regulate F-actin stability and dynamics. Loss of 14-3-3 $\lambda$ in Arabidopsis resulted in longer etiolated hypocotyls in dark and changed actin cytoskeleton architecture in hypocotyl cells. Overexpression of $A D F 1$ repressed 14-3-3 $\lambda$ mutant hypocotyl elongation and actin dynamic phenotype. In addition, the phosphorylation level of ADF1 was increased and the subcellular localization of ADF1 was altered in 14-3-3 $\lambda$ mutant. Consistent with these observations, the actin filaments were more stable in 14-3-3 $\lambda$ mutant. Our results indicate that 14-3-3 $\lambda$ protein mediates F-actin dynamics possibly through inhibiting ADF1 phosphorylation in vivo.
\end{abstract}

Arabidopsis, 14-3-3, ADF, phosphorylation, actin cytoskeleton dynamics

Citation: Zhao SS, Zhao YX, Guo Y. 14-3-3 $\lambda$ protein interacts with ADF1 to regulate actin cytoskeleton dynamics in Arabidopsis. Sci China Life Sci, 2015, 58: 1142-1150, doi: 10.1007/s11427-015-4897-1

As one of major cytoskeleton networks, actin filaments constantly undergo rapid reorganization in plant cells, which is required for variety of cellular processes ranging from cell division, morphogenesis, elongation, tip growth and cell movement and plays an important role in plant growth, development and stress responses [1-6]. The reorganization of actin filaments is regulated by an array of actin binding proteins, such as monomer binding protein profilin, side binding protein fimbrin, actin bundling protein villin, and actin depolymerizing factor (ADF) [1,2,7-9]. ADF proteins have ability to bind both G-actin monomer and F-actin filament, and modulate actin filament reorganization through depolymerizing filaments from their pointed ends, severing actin filaments, and increasing the concentra-

*Corresponding author (email: zhaoyx@ @sdnu.edu.cn; guoyan@cau.edu.cn) tion of barbed ends for promotion of actin polymerization [8-12]. The ADF family contains 11 members in Arabidopsis. They are divided into four subclasses [13,14]. These protein members contribute to F-actin turnover process during pollen tube elongation, hypocotyl cell growth, trichome development, and stomatal movement [15-17]. $A D F 1, A D F 2, A D F 3$, and $A D F 4$ are subclass I members and constitutively express in whole plant except pollen. Loss of $A D F 1$ results in bundled actin arrays in hypocotyl and overexpression of $A D F 1$ causes actin filament instable [17]. The activity of ADF is regulated by many factors such as $\mathrm{pH}$, phosphoinositide 4,5-bisphosphate (PIP2) and phosphorylation [18]. The phosphorylation of Ser 6 is required for ADF actin binding and severing activity [18,19]. Phosphorylation is key regulatory mechanism of ADF activity [20,21]. However, these regulation pathways are 
not well documented in plant. In Arabidopsis, ADF1 is possibly phosphorylated by calcium-dependent protein kinase (CDPK) at Ser $6[19,20]$.

14-3-3 proteins are highly conserved and ubiquitous in eukaryotes, and are adaptor proteins interacting with phosphorylated proteins at their phosphorylation site [22]. In Arabidopsis, 14-3-3 protein family contains 13 members that interact with various targets involved in regulation of plant growth and development, abiotic and biotic stress responses $[23,24]$. Previous studies demonstrate that 14-3-3 $\lambda$ and $\kappa$ proteins interact with and repress salt overly sensitive 2 (SOS2) activity in the absence of salt stress. The interaction between these 14-3-3 proteins and SOS2 is reduced when plant responds to salt stress in order to activating SOS pathway for salt tolerance [24]. The expression of 14-3-3 $p s i$ is specific induced by low temperature [25,26]. 14-3-3 psi negatively regulates plant freezing tolerance by modulating cold-induced gene expression [27]. In animals, 14-3-3 proteins indirectly regulate actin cytoskeleton assembly and disassembly through interacting with phosphorylated cofilin/ADF [28]. 14-3-3 proteins also interact with and inhibit the kinases LIM kinsase (LIMK) and testicular protein kinase (TESK) which are responsible for cofilin phosphorylation [29,30]. In animals, the cofilin-phosphatase SSH1 stimulates F-actin disassembly by desphosphorylating cofilin. 14-3-3 interacts with slingshot homolog 1 (SSH1) and hinders its cofilin-phosphatase activity. Overexpression of 14-3-3 inhibits cofilin dephosphorylation [31,32]. 14-3-3 regulates F-actin turnover by altering cofilin-phosphorylation in animals.

However in plant, it is not known if 14-3-3 proteins are involved in regulation of F-actin assembly and disassembly. In this study, we found that 14-3-3 $\lambda$ inhibits ADF1 phosphorylation in vivo to regulate F-actin dynamics.

\section{Materials and methods}

\subsection{Plant materials and growth conditions}

Arabidopsis thaliana ecotype Columbia (Col-0) was used as the wild type in this study. Arabidopsis thaliana T-DNA insertion line, 14-3-3 $\lambda$ (Salk_075219) with an insertion in the second intron of At5g10450 was obtained from ABRC and identified by PCR with primers $14-3-3 \lambda \mathrm{F}$ (5'-AGCCCACCTGACTCTTTGCC-3'), 14-3-3 $\lambda$ R (5'-ATCCGCAGCTGCGATATCCT-3') and T-DNA left border primer LBa 1 (5'-TGGTTCACGTAGTGGGCCATCG-3'). The mutant was reconfirmed by RT-PCR, with the primer 14-3-3 $\lambda$ CDS F (5'-ATGGCGGCGACATTAGGCAG-3') and 14-3-3 $\lambda$ CDS R (5'-TCAGGCCTCGTCCATCT-3'). T-DNA insertion line adfl (Salk_144459) was kindly provided by Prof. Huang ShanJin (Institute of Botany, Chinese Academy of Sciences, China, [33]) and identified with primers ADFl F (5'-GAGCTCGCGTAGATCATCTTG-3'),
ADFl R (5'-CGCCCGAAGAAATATCTTCTC-3') and LBa1. To generate 14-3-3 $\lambda$ adfl double mutant, adfl was crossed into 14-3-3 $\lambda$. All the mutants and transgenic plants are in Col-0 background.

\subsection{Scanning electron microscopy assay}

To observe the hypocotyl epidermal cells, 6-day-old seedlings of Col-0 and 14-3-3 $\lambda$ mutant grown in dark were used for scanning electron microscopy assay. Hypocotyls were separated from seedlings and fixed with double sided adhesive type. Scanning electron microscope Hitachi TM3000 (Hitachi, Japan) was used. Magnification is $100 \times$ under the microscope. One hundred hypocotyl epidermal cells were taken for the cell length measurement using ImageJ software (http://rsb.info. nih.gov/ij/).

\subsection{Fluorescence microscopy of actin filaments}

To visualize the actin filament dynamics in vivo, we transformed construct Pro35S:GFP(green fluorescent protein)-fABD2-GFP into Col-0, 14-3-3 $\lambda$, adf1 and 14-3-3 $\lambda$ adfl background. For the observation of actin filaments in transgenic plants harboring Pro35S:ADF1 or Pro35S: $6 \times M y c-14-3-3 \lambda$ in 14-3-3 $\lambda$ background, we crossed these transgenic lines into Col-0 expressing GFP-fABD2-GFP reporter. The homozygotes were identified in $\mathrm{F}_{2}$ population. Seeds were sterilized and stratified for $3 \mathrm{~d}$ at $4^{\circ} \mathrm{C}$ on $0.5 \times$ Murashige and Skoog (MS) medium with $1 \%$ sucrose supplemented, and exposed to light for $6 \mathrm{~h}$ before wrapped in aluminum foil for dark treatment. Actin filaments were observed by a confocal laser scanning microscope Leica TCS SP5 (Leica, Germany), equipped with a $60 \times$ oil objective. GFP signal was excited at $488 \mathrm{~nm}$.

For in vitro actin staining assay, F-actin was preformed using $5 \mu \mathrm{mol} \mathrm{L}{ }^{-1}$ of $\mathrm{G}$-actin protein incubated at $25^{\circ} \mathrm{C}$ for $30 \mathrm{~min}$ in a polymerization buffer $\left(10 \mathrm{mmol} \mathrm{L}^{-1}\right.$ imidazole, $\mathrm{pH}$ 7.0, 2 mmol L${ }^{-1} \mathrm{MgCl}_{2}, 0.2 \mathrm{mmol} \mathrm{L}{ }^{-1}$ ATP, $50 \mathrm{mmol} \mathrm{L}^{-1}$ $\mathrm{KCl}, 1 \mathrm{mmol} \mathrm{L}{ }^{-1}$ ethylene glycol tetraacetic acid (EGTA), $0.2 \mathrm{mmol} \mathrm{L}^{-1} \mathrm{CaCl}_{2}, 0.5 \mathrm{mmol} \mathrm{L}^{-1}$ dithiothreitol (DTT), and $3 \mathrm{mmol} \mathrm{L}^{-1} \mathrm{NaN}_{3}$ ). Preformed actin filaments were then incubated with the $14-3-3 \lambda$ protein at $25^{\circ} \mathrm{C}$ for $30 \mathrm{~min}$. An equimolar amount of Alexa-488-phalloidin (Invitrogen, USA) was added to the labeled actin filaments for $15 \mathrm{~min}$ at $25^{\circ} \mathrm{C}$. Prestained actin filaments were diluted to $10 \mathrm{nmol} \mathrm{L}{ }^{-1}$ to be observed with an Andor iXon charge-coupled device camera (Andor, UK).

\subsection{Quantitative analysis of actin filament array in hypocotyl cells}

The measurement of skewness and density of filament was described by Higaki et al. [34]. Skewness was measured to quantify the extent of filament bundling. Filament density 
was calculated as the percent occupancy of GFP-fABD2GFP signal in each micrograph of hypocotyl cells. One hundred hypocotyl cells were selected out from 20 individual seedlings of each genotype and used for the measurement. The micrographs of the hypocotyl cells were analyzed in ImageJ with plugins of skewness and density.

\subsection{Coimmunoprecipitation assay in Arabidopsis leaf protoplasts}

For coimmunoprecipitaion assay, 14-3-3 $\lambda$ coding region was amplified and cloned into $p C A M B I A 1307-6 \times M y c$ vector between $X b a \mathrm{I}$ and $S a l \mathrm{I}$ sites. $A D F 1$ coding region was cloned into $p$ CAMBIA1205-3×Flag vector between BamHI and $E c o R I$ sites. The resultant plasmids were cotransformed into wild-type protoplasts. After incubation at $23^{\circ} \mathrm{C}$ overnight, total protein was extracted from the protoplasts using $2 \mathrm{~mL}$ immunopreciptitation buffer $\left(10 \mathrm{mmol} \mathrm{L} \mathrm{L}^{-1}\right.$ Tris, $\mathrm{pH}$ $7.5,0.5 \%$ Nonidet P-40, $2 \mathrm{mmol} \mathrm{L}^{-1}$ eathylene diamine tetraacetic acid (EDTA), $150 \mathrm{mmol} \mathrm{L}^{-1} \mathrm{NaCl}, 1 \mathrm{mmol} \mathrm{L}^{-1}$ phenylmethanesulfonyl fluoride (PMSF), and $1 \%$ protease inhibitor cocktail (Sigma-Aldrich, USA)). Myc-14-3-3 $\lambda$ protein was purified with $30 \mu \mathrm{L}$ anti-Myc agarose (Sigma-Aldrich) from the total protein. After washed with $2 \mathrm{~mL}$ immunopreciptitation buffer for three times, the agarose was used for the western blot assays with anti-Flag antibody. Protoplasts only expressing pCAMBIA1205-3×Flag$A D F 1$ were used as a negative control.

\subsection{Firefly luciferase complementation assay in tobacco}

For the split luciferase assay, the $14-3-3 \lambda$ and $A D F 1$ cDNA coding region was amplified and cloned into the KpnI and SalI sites of $p C M 1307-n L U C$ and $p C M 1307-c L U C$ vector, respectively. The plasmids were introduced into Agrobacterium tumefaciens GV3101 and co-infiltrated into the leaves of 3-week-old Nicotiana benthamiana. After 3-day incubation, the LUC activity was measured by cooled charge coupled device (CCD) imaging camera (1300B; Roper, Germany). $1 \mathrm{mmol}^{-1}$ luciferin as substrate of luciferase was sprayed onto the leaves.

\subsection{Immunoblot analysis with anti-phosphor-Ser/Thr antibody}

Pro35S:3 $\times$ Flag-ADF1 was transformed into wild type and 14-3-3 $\lambda$ mutant, respectively. Total protein was isolated from the resultant transgenic seedlings in a extraction buffer (10 mmol L ${ }^{-1}$ Tris, pH 7.5, 0.5\% Nonidet P-40, 2 mmol L ${ }^{-1}$ EDTA, $150 \mathrm{mmol} \mathrm{L}^{-1} \mathrm{NaCl}, 1 \mathrm{mmol} \mathrm{L}{ }^{-1} \mathrm{PMSF}$, and $1 \%$ protease inhibitor cocktail (Sigma-Aldrich)). ADF1 protein was immunopreciptitated from the total protein by incubating with anti-Flag agarose. For the phosphatase treatment, the immunopreciptitated protein was treated with $\lambda$ phosphatase (Promega, USA) at $30^{\circ} \mathrm{C}$ for $15 \mathrm{~min}$ [35]. Im- munoblotting was performed with anti-Flag antibody or anti-phosphor-Ser/Thr antibody (Sigma-Aldrich).

\subsection{High speed cosedimentation assay}

The high speed cosedimentation assays were performed according to Kovar et al. [36] and Huang et al. [37]. Actin was purified as previously published method according to Spudich and Watt [38]. Recombinant protein His-14-3-3 $\lambda$ was purified with Ni-NTA agarose eluted from agarose. The purified His-14-3-3 $\lambda$ and G-actin were preclarified at $150,000 \times g$ for $30 \mathrm{~min}$ at $4^{\circ} \mathrm{C}$. $2 \mu \mathrm{mol} \mathrm{L}{ }^{-1} 14-3-3 \lambda$ were incubated with $4 \mu \mathrm{mol} \mathrm{L}{ }^{-1}$ preformed F-actin for $30 \mathrm{~min}$ at $25^{\circ} \mathrm{C}$. The reaction mixture was centrifuged at $150,000 \times g$ for $30 \mathrm{~min}$ at $4^{\circ} \mathrm{C}$. The supernatant and pellet were used for SDS-PAGE analysis and Commassie Brilliant Blue R250 staining. The relative amount of F-actin in the supernatant and pellet was quantified by densitometry in ImageJ software.

\section{Results}

\subsection{4-3-3 $\lambda$ mutant exhibits longer etiolated hypocot- yls in dark}

We performed a genetic screen for mutants that exhibit longer etiolated hypocotyls in dark from T-DNA insertion lines ordered from the Arabidopsis Biological Resource Center. Seeds were exposed to light for $6 \mathrm{~h}$ before wrapped in aluminum foil for 4-, 6- or 8-day dark treatment. A mutant 14-3-3 $\lambda$ (Salk_075219) was identified with longer hypocotyl under dark (Figure 1A and B). Scanning electron microscopy analysis of hypocotyls from 6-day-old seedlings grown in dark revealed that the average length of hypocotyl cells was significantly increased in 14-3-3 $\lambda$ compared with that of wild type (Figure 1C and D). Under light condition, the hypocotyl length in 14-3-3 $\lambda$ was also slightly longer than that of Col-0 (Figure $1 \mathrm{E}$ and F). We previously published that $14-3-3 \lambda$ is a regulator of SOS2 kinase [24]. Consistent with our previous results, the homozygous 14-3-3 $\lambda$ mutant contained a T-DNA insertion in the 2nd intron of At5g10450, and a very low amount of transcript was detected by RT-PCR in the mutant plants (Figure $1 \mathrm{G}$ and $\mathrm{H}$ ). To rescue the 14-3-3 $\lambda$ mutant hypocotyl elongation phenotype, we generated the transgenic lines expressing Pro35S:14-3-3 $\lambda$ in 14-3-3 $\lambda$ mutant background (Figure $1 \mathrm{H})$. The hypocotyl elongation phenotype was rescued in the transgenic lines (Figure 1A and B), suggesting that the phenotype is indeed caused by the mutation in 14-3-3 $\lambda$.

In our previous study, the $14-3-3 \lambda-G U S$ expression patterns and the immunoblot assays with 14-3-3 $\lambda$ antibodies indicate that 14-3-3 $\lambda$ protein ubiquitously expresses in all tissues including hypocotyl [24]. 

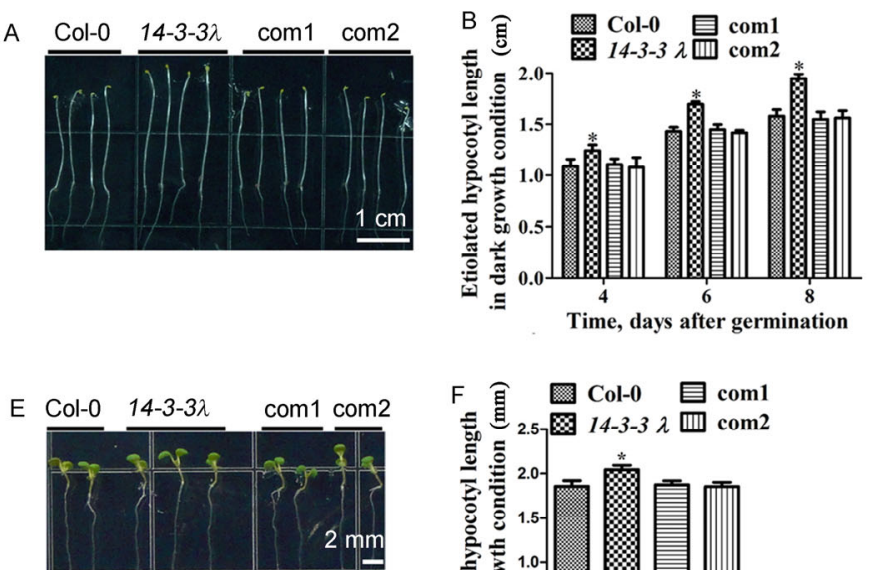
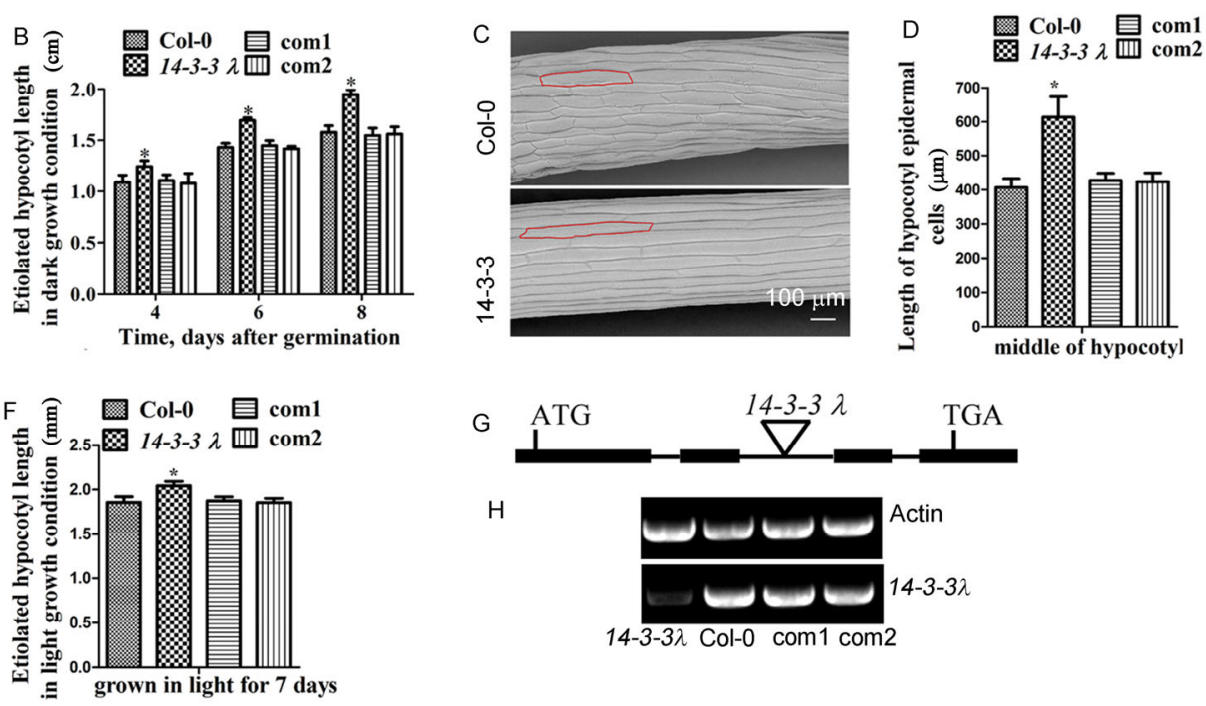

Figure 1 14-3-3 $\lambda$ mutant seedlings have longer etiolated hypocotyls in dark growth condition. A, Dark-grown 14-3-3 $\lambda$ mutant seedlings exhibit longer etiolated hypocotyls than that of wild type. The phenotype is rescued by expressing 14-3-3 $\lambda$. B, Etiolated hypocotyl length measurement of wild type, 14-3-3 $\lambda$ mutant and two rescued transgenic lines in dark growth condition. Significant differences were observed between 14-3-3 $\lambda$ and Col-0. Error bars represent $\bar{x} \pm \mathrm{SD}, n=100$. Statistical significance was determined by Student's $t$ test; significant differences $(*, P<0.05)$. Two rescued transgenic lines, com 1 and com2. C, Scanning electron microscopy images of dark-grown hypocotyl epidermal cells from Col-0, 14-3-3 $\lambda$. D, Length of hypocotyl epidermal cells of Col-0, 14-3-3 $\lambda$ and two rescued lines grown in dark for $6 \mathrm{~d}$. Error bars represent $\bar{x} \pm \mathrm{SD}$, Student's $t$ test, * $P<0.05, n=100$. E, $14-3-3 \lambda$ mutant seedlings exhibit longer etiolated hypocotyls than wild type in light growth condition. F, Etiolated hypocotyl length measurement of Col-0, 14-3-3 $\lambda$ and two rescued lines grown in light for $7 \mathrm{~d}$. Error bars represent $\bar{x} \pm \mathrm{SD}$, Student's $t$ test, *, $P<0.05$. G, Structure of $14-3-3 \lambda$ gene. The filled black boxes indicated exons, and the lines between the boxes indicate introns. H, 14-3-3 $\lambda$ is a knockdown allele. RT-PCR analysis showed a deficient expression of $14-3-3 \lambda$ gene in 14-3-3 $\lambda$ mutant. 14-3-3 $\lambda$ expression was restored in the two rescued lines.

\section{$2.2 \quad$ 14-3-3 $\lambda$ mutant shows impaired actin stability and changed actin architecture in hypocotyls}

Actin cytoskeleton dynamics plays a crucial role during hypocotyl elongation. To determine whether the altered hypocotyl elongation in 14-3-3 $\lambda$ mutant is due to abnormal actin cytoskeleton dynamics, we observed actin dynamics in hypocotyl cells of 14-3-3 $\lambda$ mutant and Col-0. To do this, the transgenic plants harboring the construct 35S:GFP$f A B D 2-G F P$ (for the second actin binding domain of At-Fim1) in Col-0 and 14-3-3 $\lambda$ background were obtained. We observed the actin structure of middle hypocotyl cells at the place about $5 \mathrm{~mm}$ distance from the root using the plants grown under dark for $5 \mathrm{~d}$ (Figure 2A). Skewness and density were used as two statistical parameters to quantify the extent of actin filament bundling and cytoskeleton density (Figure 2B and C). Actin arrays altered in 14-3-3 $\lambda$ mutant hypocotyl epidermal cells. Skewness of actin cytoskeleton increased in 14-3-3 $\lambda$ mutant compared with wild type. The density of actin filaments decreased in 14-3-3 $\lambda$ mutant compared with wild type. Actin filaments in 14-3-3 $\lambda$ mutant turned more bundled and less dense than that in wild type.

To determine if $14-3-3 \lambda$ is required for F-actin stability, the transgenic lines were treated with $200 \mathrm{nmol} \mathrm{L}^{-1}$ Lat A for $45 \mathrm{~min}$, the actin filaments became more fragmented and less abundant in both Col-0 and 14-3-3 $\lambda$ hypocotyl cells compared with that in untreated hypocotyl cells (Figure 2D). However, the actin filaments in 14-3-3 $\lambda$ hypocotyl cells appeared less fragmented than that in Col-0 (Figure 2D). The actin filament shortening was detected in $80 \%$ of the cells in Col-0. By contrast, the actin network was slightly affected in $50 \%$ of cells in 14-3-3 $\lambda$ mutant (Figure 2E).

Taken together, mutation in $14-3-3 \lambda$ resulted in actin stability increased and actin architecture altered in the hypocotyl cells at dark condition. These results suggest that 14-3-3 $\lambda$ is involved in regulation of F-actin reorganization in the hypocotyl cells under dark condition.

\subsection{4-3-3 $\lambda$ does not bind to actin filaments and has no effect on actin polymerizing}

To further determine whether 14-3-3 $\lambda$ directly regulates actin reorganization, an actin cosedimentation assay at high speed centrifugation was performed (Figure S1A-C). 14-3-3 $\lambda$ was failed to cosediment with actin filaments, and 14-3-3 $\lambda$ did not affect the amount of actin in the supernatants. Stomatal closure-related actin binding protein 1 (SCAB1), an actin binding and bundling protein [3], was used as a positive control. The high speed cosedimentation results indicate that $14-3-3 \lambda$ has no actin filament binding activity in vitro.

To further confirm this conclusion, we preformed fluo- 

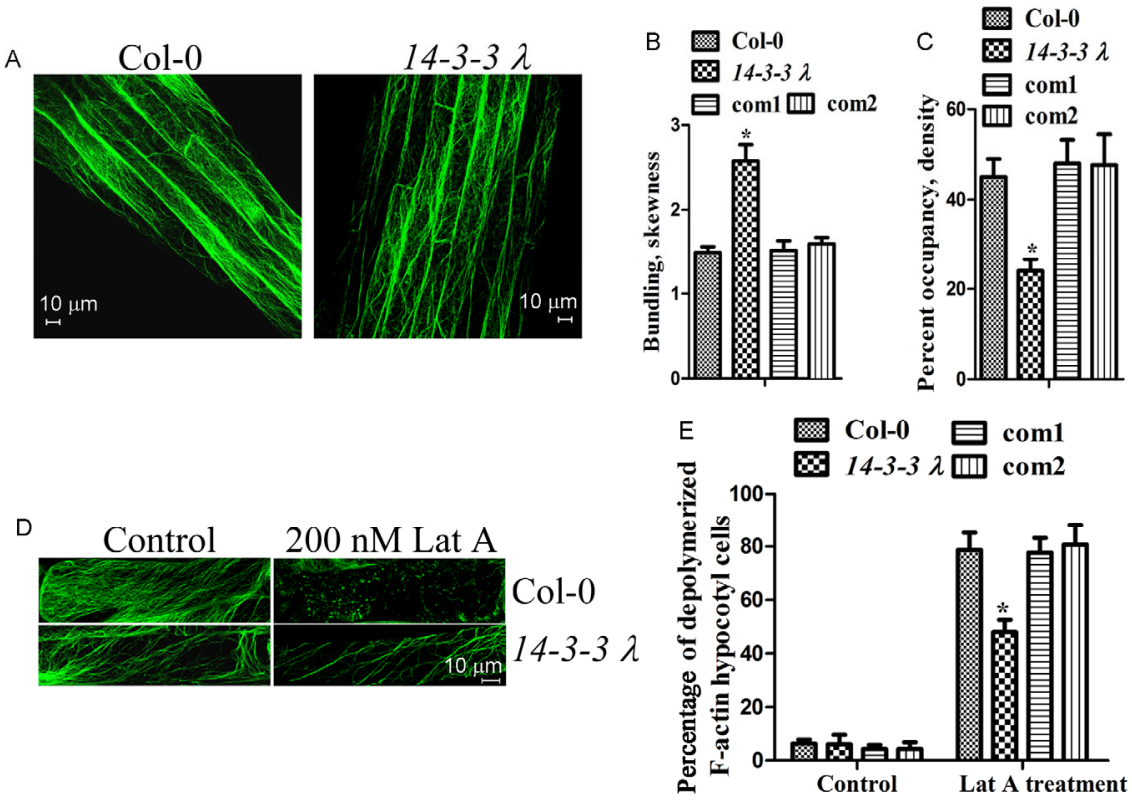

Figure 2 Architecture of actin cytoskeleton is altered in hypocotyl epidermal cells of 14-3-3 $\lambda$ seedlings. A, Architecture of actin cytoskeleton in dark grown etiolated hypocotyl epidermal cells of Col-0, and 14-3-3 $\lambda$ seedlings. B, The extent of filament bundling (skewness) of hypocotyl cells from Col-0, 14-3-3 $\lambda$ mutant and two rescued transgenic lines com1 and com2. C, Average filament density of hypocotyl cells from Col-0, 14-3-3 $\lambda$ mutant and two rescued transgenic lines com 1 and com 2 . D, Actin filament organization of hypocotyl cells grown in dark for $5 \mathrm{~d}$ before and after 200 nmol $\mathrm{L}^{-1}$ Lat A treatment from Col-0, 14-3-3 $\lambda$ mutant and two rescued lines. E, Percentage of hypocotyl cells with depolymerized actin filaments after Lat A treatment from Col-0, 14-3-3 $\lambda$, and two rescued lines in 14-3-3 $\lambda$. Values of B-E represent $\bar{x} \pm \mathrm{SD}, n=50$. Statistical significance was determined by Student's $t$ test; significant differences $(*, P<0.01)$.

rescence light microscopy assay. Preformed F-actin was incubated with $14-3-3 \lambda$ protein at $25^{\circ} \mathrm{C}$ for $30 \mathrm{~min}$ and then stained with Alexa-488-phalloidin for $15 \mathrm{~min}$. The SCAB1 was also used as a positive control. Consistently, fluorescence light microscopy assay revealed that $14-3-3 \lambda$ neither inhibits nor promotes actin polymerization. As a control, SCAB1 bundled actin filaments.

These results suggest that $14-3-3 \lambda$ protein does not bind to actin filaments directly and has no effect on actin polymerization or depolymerization in vitro.

\subsection{4-3-3 $\lambda$ interacts with ADF1 in vivo}

Because 14-3-3 $\lambda$ does not bind to actin filaments directly, we proposed that $14-3-3 \lambda$ interacts with an actin associated or binding protein to indirectly regulate actin reorganization in vivo. In animal, it has been shown that 14-3-3 proteins colocalized with and interacts with F-actin depolymerizing and severing factor ADF/cofilin proteins [39]. From bovine brain, cytosol 14-3-3 $\zeta$ is identified by Mass Spectromety as a phosphocofilin binding protein to stablize phosphotylated cofilin and regulate actin dynamics [28]. In plant, ADF1 and ADF4 are reported as regulators involved in hypocotyl elongation $[15,17]$. To determine the relationship between 14-3-3 $\lambda$ and ADF proteins, we performed coimmunoprecipitation assay to detect the interaction between 14-3-3 $\lambda$ and ADF1 (Figure 3A). Myc-14-3-3 $\lambda$ protein was purified by anti-Myc agarose using total protein extract from the protoplast coexpressing Pro35S:3 $\times$ Flag-ADF1 and Pro35S:6×Myc-14-3-3 $\lambda$. Immunobloting assay was performed using anti-Flag antibody to detect Flag-ADF1 in the precipitation products. 14-3-3 $\lambda$ pulled down ADF1. As a control, the anti-Myc agarose did not pulled down the Flag-ADF1. Coimmunoprecipitation results indicate that 14-3-3 $\lambda$ interacts with ADF1 in vivo.

To further confirm the interaction between 14-3-3 $\lambda$ and ADF1, split-luciferase (split-LUC) complementation assay in Nicotiana benthaminana was performed. ADF1 and 14-3-3 $\lambda$ were fused to the $\mathrm{C}$ terminus and $\mathrm{N}$ terminus of LUCIFERASE, respectively. The resulted plasmids were cotransformed into Nicotiana benthaminana leaves. To measure luciferase activity, the cooled CCD image apparatus was used to capture the LUC image. Relative LUC activity per $\mathrm{cm}^{2}$ infiltrated leaf area was calculated. The split-LUC assays showed that transient co-expression of nLUC-14-3-3 $\lambda$ and ADF1-cLUC in $N$. benthamiana yielded strong fluorescence signals, but no fluorescence signal was detected in the control leaves which co-expressing nLUC-14-3-3 $\lambda$ and cLUC or nLUC and ADF1-cLUC, which further confirms that 14-3-3 $\lambda$ interacts with ADF1 in vivo (Figure 3B).

To further evaluate whether 14-3-3 $\lambda$ affects ADF1 F-actin binding and depolymerizing activity, high speed cosedimentation assay was performed (Figure S2). $2 \mu \mathrm{mol} \mathrm{L} \mathrm{L}^{-1}$ 


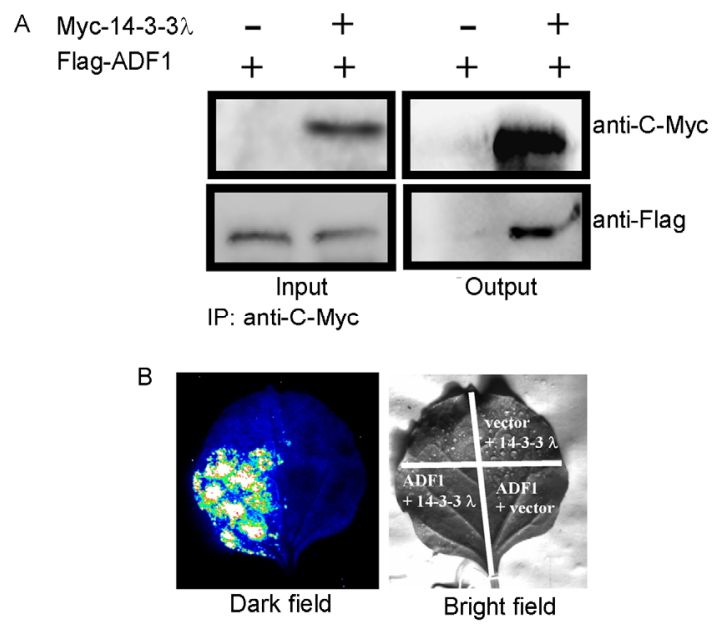

Figure 3 14-3-3 $\lambda$ interacts with ADF1 in vivo. A, Coimmunoprecipitation of 14-3-3 $\lambda$ with ADF1. Arabidopsis protoplasts transiently expressing Flag-ADF1 or coexpressing Myc-14-3-3 $\lambda$ and Flag-ADF1 were immunoprecipitated with anti-C-Myc agarose. Immunoblot was probed with anti-Myc or anti-Flag antibody. B, Split luciferase complementation imaging assay in Nicotiana benthamiana.

of ADF1 protein was incubated with $2 \mu \mathrm{mol} \mathrm{L}{ }^{-1}$ His-14-3-3 $\lambda$ protein and $4 \mu \mathrm{mol} \mathrm{L} \mathrm{L}^{-1}$ preformed $\mathrm{F}$-actin at $25^{\circ} \mathrm{C}$ for 30 min. As a control, $2 \mu \mathrm{mol} \mathrm{L}{ }^{-1} \mathrm{ADF} 1$ protein was incubated with $4 \mu \mathrm{mol} \mathrm{L}{ }^{-1}$ preformed F-actin at $25^{\circ} \mathrm{C}$ for $30 \mathrm{~min}$. Consistent with ADF1 depolymerizing function, the amount of actin in the supernatant was increased when ADF1 was added in the reaction. However addition of 14-3-3 $\lambda$ did not lead to any obvious change of actin amount in the supernatant. These results suggest that $14-3-3 \lambda$ has no effect on ADF1 F-actin binding and depolymerizing activity.

\subsection{4-3-3 $\lambda$ is required for ADF1 phosphorylation and subcellular localization}

Since 14-3-3 $\lambda$ did not affect the ADF1 F-actin depolymerizing activity in vitro, we proposed $14-3-3 \lambda$ may be involved in the regulation of ADF1 F-actin depolymerizing activity in vivo. ADF/cofilin activity is regulated by phosphorylation of Ser 6 in plants and Ser 3 in animals. Basis of these studies, we prospected that 14-3-3 $\lambda$ may be involved in $\mathrm{ADF} 1$ phosphorylation regulation in vivo. To determine the phosphorylation level of ADF1 in 14-3-3 $\lambda$ mutant, we performed Western blot assay using commercial anti-phospho-serine/theronine antibodies (Figure 4A). Transgenic plants expressing Pro35S:3 $\times$ Flag-ADF1 in Col-0 and 14-3-3 $\lambda$ background were obtained. Flag-ADF1 protein was purified with anti-Flag agarose from the transgenic plant seedlings. As a control, the immunoprecipitated proteins from wild-type seedlings on the Flag-agarose were treated with Lambda Protein Phosphatase ( $\lambda$ phosphatase, NEW ENGLAND BioLabs). Western blot assay was performed using the pull-down product on the Flag agarose.

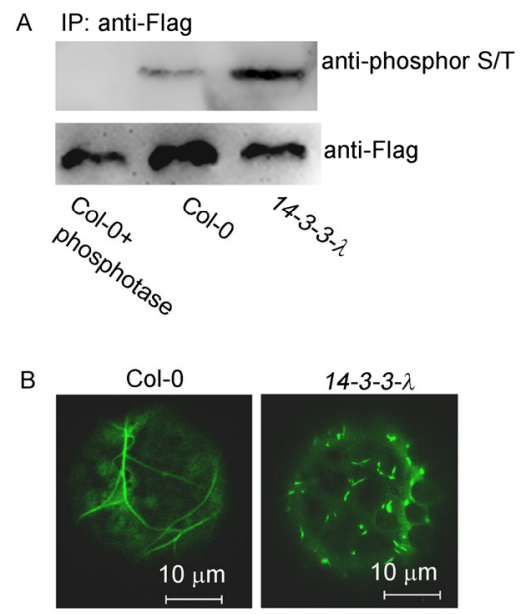

Figure $4 \quad 14-3-3 \lambda$ is required for ADF1 phosphorylation and subcellular localization. A, Western blot assay with anti-phospho Serine/Theronine antibody. Flag-ADF1 was immunoprecipitated with anti-Flag agarose from transgenic plants expressing Pro35S:3 $\times$ Flag-ADF1 in Col-0 or 14-3-3 $\lambda$ background. B, ADF1 localization was altered in 14-3-3 $\lambda$ mutant. GFP-ADF1 was transformed into protoplasts isolated from Col-0 or 14-3-3 $\lambda$ for confocal imaging.

The phosphorylation level of ADF1 was nearly abolished after Lambda Protein Phosphatase treatment, and the phosphorylation level of ADF1 in 14-3-3 $\lambda$ mutant was increased compared with that in wild type. These results suggest that $14-3-3 \lambda$ is involved in ADF1 phospho-regulation in vivo.

Phosphorylation of ADF at Ser 6 in plant affects ADF actin binding activity and alters ADF subcellular localization in vivo $[12,19]$. It has been shown that ADF1 subcellular localization is associated with its phosphorylation level in protoplast [40]. Since 14-3-3 $\lambda$ is involved in ADF1 phosphorylation regulation, we proposed that $14-3-3 \lambda$ may affect ADF1 subcellular localization. To determine this hypothesis, we constructed ProUBQ10:GFP-ADF1 and transiently expressed this construct in protoplasts of Col- 0 and 14-3-3 $\lambda$ mutant (Figure 4B). More than 100 protoplasts from each genotype were used for the fluorescence observation. In wild type, the GFP-ADF1 was detected as the structure of filaments in $85 \%$ protoplasts. However, the GFP-ADF1 was observed as smear or fragmented filaments in cytosol in $70 \%$ protoplasts of $14-3-3 \lambda$ mutant. These results suggest that $14-3-3 \lambda$ is required for ADF1 proper localization, possibly by affecting phosphorylation status of ADF1.

\subsection{Genetic interaction between 14-3-3 $\lambda$ and ADF1}

To confirm the interaction relationship in genetic level, we generated 14-3-3 $\lambda$ adf1 double mutant by crossing an adf1 T-DNA insertion (Salk_144459) into 14-3-3 $\lambda$. The Col-0, 14-3-3 $\lambda$, adfl, and 14-3-3 $\lambda$ adf1 were used for dark-grown 

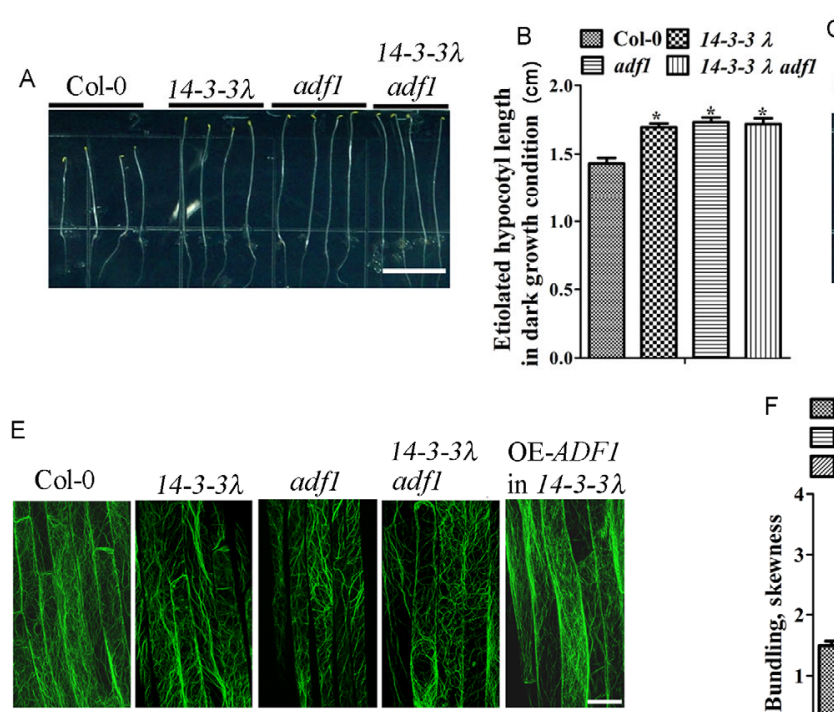
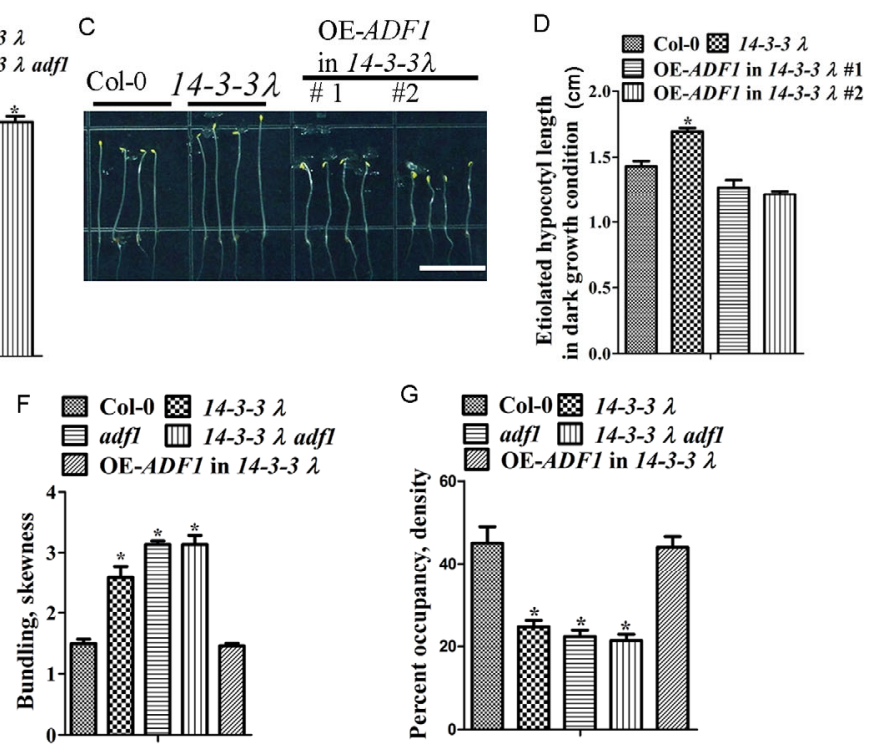

Figure 5 Genetic interaction between 14-3-3 $\lambda$ and ADF1. A, Dark-grown Hypocotyl phenotype of Col-0, 14-3-3 $\lambda$, adf1, and 14-3-3 $\lambda$ adf1 double mutant. B, Etiolated hypocotyl length measurement of Col-0, 14-3-3 $\lambda$, adf1, and 14-3-3 $\lambda$ adf1. C, Dark-grown hypocotyl phenotype in Col-0, 14-3-3 $\lambda$, and transgenic lines overexpressing $A D F 1$ in 14-3-3 $\lambda$. D, Etiolated hypocotyl length measurement of Col-0, 14-3-3 $\lambda$, and transgenic lines overexpressing $A D F 1$ in 14-3-3 $\lambda$. E, Architecture of actin cytoskeleton in dark grown etiolated hypocotyl epidermal cells of Col-0, 14-3-3 $\lambda$, adf1, 14-3-3 $\lambda$ adf1 and ADF1 overexpression transgenic line in 14-3-3 $\lambda$. F, The extent of actin filament bundling (skewness) of hypocotyl cells shown in E. G, Average of actin filament density of hypocotyl cells shown in E. Difference is significant and values in B, D, F, and G represent $\bar{x} \pm \mathrm{SD}, n=50$. Statistical significance was determined by Student's $t$ test; significant differences $(*, P<0.05)$.

hypocotyl elongation assay (Figure 5A and B). RT-PCR assay showed that adfl is a knock out line (Figure S3A). Similar to the 14-3-3 $\lambda$ mutant, adf1 mutant showed longer hypocotyls compared to wild type under dark treatment. This is consistent with the previous report that loss of $A D F 1$ resulted in significant increase of hypocotyl length [17]. 14-3-3 $\lambda$ adf1 double mutant showed a similar hypocotyl elongation phenotype as 14-3-3 $\lambda$ and $a d f 1$ mutants (Figure $5 \mathrm{~A}$ and $\mathrm{B})$. We obtained $A D F 1$ overexpression transgenic lines harboring Pro35S:ADF1 in 14-3-3 $\lambda$ mutant background. RT-PCR results showed the expression level of $A D F 1$ is increased in these transgenic lines (Figure S3B). Overexpression $A D F 1$ in 14-3-3 $\lambda$ mutant resulted in obviously decreased hypocotyl length compared to wild type (Figure 5C and D).

The F-actin array was also observed in hypocotyl epidermal cells in Col-0, 14-3-3 $\lambda$, adf1, 14-3-3 $\lambda$ adfl and $A D F 1$ overexpression transgenic lines in 14-3-3 $\lambda$ mutant background (Figure 5E). Actin filaments of hypocotyl cells in 14-3-3 $\lambda, a d f 1$, and 14-3-3 $\lambda$ adf1 were more bundled and less dense than that in Col-0 (Figure 5F and G). Overexpression $A D F 1$ in 14-3-3 $\lambda$ rescued its F-actin architecture phenotype (Figure $5 \mathrm{~F}$ and $\mathrm{G}$ ). These results suggest that 14-3-3 $\lambda$ genetically interacts with ADF1.

\section{Discussion}

Actin cytoskeleton undergoes constantly reorganization through polymerizing and depolymerizing, which plays essential roles in plant growth and development. Various proteins including actin associated factors or actin binding proteins involved in regulation of actin dynamics. Actin binding proteins directly bind actin and have effect on actin polymerizing or depolymerizing. ADF has ability to bind F-actin, and functions directly in F-actin depolymerizing and severing process. In plant, actin interacting proteins (AIP) interact with ADF proteins and enhance their F-actin severing and depolymerizing activity [41-43]. The activity of ADF proteins is also regulated by phosphorylation in plant [20]. It has been reported that CDPKs phosphorylate ADFs in plant $[19,20]$. In this study, we identify that 14-3-3 $\lambda$ interacts with ADF1 in plant and functions in ADF1 phosphorylation regulation to modulate actin cytoskeleton reorganization.

ADF plays a critical role on single actin filament turnover. Loss of ADF1 in Arabidopsis results in more bundled and less dense actin arrays in hypocotyl cells, which is associated with the longer hypocotyl elongation under dark [17,19]. ADF actin severing and depolymerizing activity is negatively regulated by phosphorylation. When ADF phosphorylation level is increased, ADF activity is reduced [12,30]. In 14-3-3 $\lambda$ mutant, ADF1 phosphorylation level was increased and its activity on actin was decreased. Actin filaments in hypocotyl cells of 14-3-3 $\lambda$ mutant showed more bundled and less dense, which is consistent with the actin array change in adfl mutant. Furthermore, overexpression of $A D F 1$ reduced 14-3-3 $\lambda$ mutant pheno- 
type. These results indicate that ADF1 genetically interacts with $14-3-3 \lambda$ and functions at downstream of 14-3-3 $\lambda$.

14-3-3 $\lambda$ regulates ADF1 activity through inhibiting ADF1 phosphorylation in vivo. However, 14-3-3 $\lambda$ did not affect ADF1 activity on actin in vitro. In animals, protein kinases LIMK and TESK phosphorylate mammalian cofilin/ADF. Plant has no such kinase homologous [44]. We speculate that $14-3-3 \lambda$ protein directly or indirectly inhibits a protein kinase, such as CDPK6, that is responsible for ADF1 phosphorylation, which then modulates ADF1 F-actin binding and depolymerizing activity to regulate hypocotyl elongation (Figure 6). However, at current stage, we do not know if 14-3-3 $\lambda$ interacts with CDPK6 kinase and represses its kinase activity. It requires for further study to understand the mechanism underlying 14-3-3-mediated regulation of actin dynamics, and find out the molecular details linking $14-3-3 \lambda$ to ADF both in vitro and in vivo.

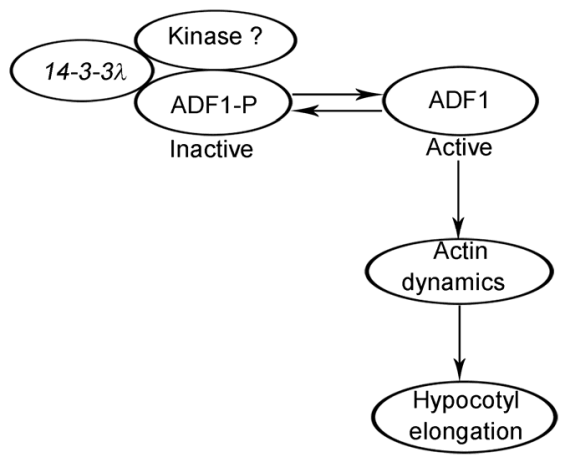

Figure 6 A working model how14-3-3 $\lambda$ regulates actin dynamics. 14-3-3 $\lambda$ interacts with and represses a kinase that is responsible for ADF1 phosphorylation, therefore to control ADF1 phosphorylation level and modulate ADF1 activity on actin dynamics.

The authors declare that they have no conflict of interest.

This work was supported by the National Basic Research Program of China (2012CB114200), and Foundation for Innovative Research Group of the National Natural Science Foundation of China (31421062).

1 Qu X, Zhang H, Xie Y, Wang J, Chen N, Huang S. Arabidopsis villins promote actin turnover at pollen tube tips and facilitate the construction of actin collars. Plant Cell, 2013, 25: 1803-1817

2 Su H, Zhu J, Cai C, Pei W, Wang J, Dong H, Ren H. FIMBRIN1 is involved in lily pollen tube growth by stabilizing the actin fringe. Plant Cell, 2012, 24: 4539-4554

3 Zhao Y, Zhao S, Mao T, Qu X, Cao W, Zhang L, Zhang W, He L, Li S, Ren S, Zhao J, Zhu G, Huang S, Ye K, Yuan M, Guo Y. The plant-specific actin binding protein SCAB1 stabilizes actin filaments and regulates stomatal movement in Arabidopsis. Plant Cell, 2011, 23: 2314-2330

4 Jiang K, Sorefan K, Deeks MJ, Bevan MW, Hussey PJ, Hetherington AM. The ARP2/3 complex mediates guard cell actin reorganization and stomatal movement in Arabidopsis. Plant Cell, 2012, 24: 2031-2040

5 Zhao Y, Pan Z, Zhang Y, Qu X, Zhang Y, Yang Y, Jiang X, Huang S, Yuan M, Schumaker KS, Guo Y. The actin-related Protein2/3 complex regulates mitochondrial-associated calcium signaling during salt stress in Arabidopsis. Plant Cell, 2013, 25: 4544-4559

6 Li M, Liu L, Xi N, Wang Y, Dong Z, Xiao X, Zhang W. Atomic force microscopy imaging of live mammalian cells. Sci China Life Sci, 2013, 56: 811-817

7 Sun T, Li S, Ren H. Profilin as a regulator of the membrane-actin cytoskeleton interface in plant cells. Front Plant Sci, 2013, 4: 512

8 Carlier MF. Control of actin dynamics. Curr Opin Cell Biol, 1998, 10: $45-51$

9 Bamburg JR. Proteins of the ADF/cofilin family: essential regulators of actin dynamics. Annu Rev Cell Dev Biol, 1999, 15: 185-230

10 Cooper JA, Schafer DA. Control of actin assembly and disassembly at filament ends. Curr Opin Cell Biol, 2000, 12: 97-103

11 Chen H, Bernstein BW, Bamburg JR. Regulating actin-filament dynamics in vivo. Trends Biochem Sci, 2000, 25: 19-23

12 Chen CY. The regulation of actin organization by actin-depolymerizing factor in elongating pollen tubes. Plant Cell, 2002, 14: 2175-2190

13 Rhee SY, Beavis W, Berardini TZ, Chen G, Dixon D, Doyle A, Garcia-Hernandez M, Huala E, Lander G, Montoya M, Miller N, Mueller LA, Mundodi S, Reiser L, Tacklind J, Weems DC, Wu Y, Xu I, Yoo D, Yoon J, Zhang P. The Arabidopsis Information Resource (TAIR): a model organism database providing a centralized, curated gateway to Arabidopsis biology, research materials and community. Nucleic Acids Res, 2003, 31: 224-228

14 Feng Y, Liu Q, Xue Q. Comparative study of rice and Arabidopsis actin-depolymerizing factors gene families. Plant Physiol, 2006, 163: 69-79

15 Henty JL, Bledsoe SW, Khurana P, Meagher RB, Day B, Blanchoin L, Staiger CJ. Arabidopsis actin depolymerizing factor 4 modulates the stochastic dynamic behavior of actin filaments in the cortical array of epidermal cells. Plant Cell, 2011, 23: 3711-3726

16 Zheng Y, Xie Y, Jiang Y, Qu X, Huang S. Arabidopsis actin-depolymerizing factor 7 severs actin filaments and regulates actin cable turnover to promote normal pollen tube growth. Plant Cell, 2013, 25: 3405-3423

17 Dong CH, Xia GX, Hong Y, Ramachandran S, Kost B, Chua NH. ADF proteins are involved in the control of flowering and regulate F-actin organization, cell expansion, and organ growth in Arabidopsis. Plant Cell, 2001, 13: 1333-1346

18 Allwood EG. Regulation of the pollen-specific actin-depolymerizing factor L1ADF1. Plant Cell, 2002, 14: 2915-2927

19 Dong CH, Hong Y. Arabidopsis CDPK6 phosphorylates ADF1 at N-terminal serine 6 predominantly. Plant Cell Rep, 2013, 32: 1715-1728

20 Smertenko AP, Jiang CJ, Simmons NJ, Weeds AG, Davies DR, Hussey PJ. Ser6 in the maize actin-depolymerizing factor, ZmADF3, is phosphorylated by a calcium-stimulated protein kinase and is essential for the control of functional activity. Plant J, 1998, 14: 187-193

21 Allwood EG, Smertenko AP, Hussey PJ. Phosphorylation of plant actin-depolymerising factor by calmodulin-like domain protein kinase. FEBS Lett, 2001, 499: 97-100

22 Li X, Dhaubhadel S. 14-3-3 proteins act as scaffolds for GmMYB62 and GmMYB176 and regulate their intracellular localization in soybean. Plant Signal Behav, 2012, 7: 965-968

23 Denison FC, Paul AL, Zupanska AK, Ferl RJ. 14-3-3 proteins in plant physiology. Semin Cell Dev Biol, 2011, 22: 720-727

24 Zhou H, Lin H, Chen S, Becker K, Yang Y, Zhao J, Kudla J, Schumaker KS, Guo Y. Inhibition of the Arabidopsis salt overly sensitive pathway by 14-3-3 proteins. Plant Cell, 2014, 26: 1166-1182

25 Jarillo JA, Capel J, Leyva A, Martinez-Zapater JM, Salinas J. Two related low-temperature-inducible genes of Arabidopsis encode proteins showing high homology to 14-3-3 proteins, a family of putative kinase regulators. Plant Mol Biol, 1994, 25: 693-704

26 Roberts MR, Salinas J, Collinge DB. 14-3-3 proteins and the response to abiotic and biotic stress. Plant Mol Biol, 2002, 50: 1031-1039

27 Catala R, Lopez-Cobollo R, Mar Castellano M, Angosto T, Alonso 
JM, Ecker JR, Salinas J. The Arabidopsis 14-3-3 protein RARE COLD INDUCIBLE 1A links low-temperature response and ethylene biosynthesis to regulate freezing tolerance and cold acclimation. Plant Cell, 2014, 26: 3326-3342

28 Gohla A, Bokoch GM. 14-3-3 regulates actin dynamics by stabilizing phosphorylated cofilin. Curr Biol, 2002, 12: 1704-1710

29 Toshima JY, Toshima J, Watanabe T, Mizuno K. Binding of 14-3-3 $\beta$ regulates the kinase activity and subcellular localization of testicular protein kinase 1. J Biol Chem, 2001, 276: 43471-43481

30 Birkenfeld J, Betz H, Roth D. Identification of cofilin and LIM-domain-containing protein kinase 1 as novel interaction partners of 14-3-3 zeta. Biolchem J, 2003, 369: 45-54

31 Nagata-Ohashi K, Ohta Y, Goto K, Chiba S, Mori R, Nishita M, Ohashi K, Kousaka K, Iwamatsu A, Niwa R, Uemura T, Mizuno K. A pathway of neuregulin-induced activation of cofilin-phosphatase Slingshot and cofilin in lamellipodia. J Cell Biol, 2004, 165: 465-471

32 Mizuno K. Signaling mechanisms and functional roles of cofilin phosphorylation and dephosphorylation. Cell Signal, 2013, 25: 457-469

33 Henty-Ridilla JL, Li J, Day B, Staiger CJ. ACTIN DEPOLYMERIZING FACTOR4 regulates actin dynamics during innate immune signaling in Arabidopsis. Plant Cell, 2014, 26: 340-352

34 Higaki T, Kutsuna N, Sano T, Kondo N, Hasezawa S. Quantification and cluster analysis of actin cytoskeletal structures in plant cells: role of actin bundling in stomatal movement during diurnal cycles in Arabidopsis guard cells. Plant J, 2010, 61: 156-165

$35 \mathrm{Lu} \mathrm{J}$, Li T, He R, Bartlett PF, Gotz J. Visualizing the microtubule-associated protein tau in the nucleus. Sci China Life Sci, 2014, 57: 422-431
36 Kovar DR, Staiger CJ, Weaver EA, McCurdy DW. AtFim1 is an actin filament crosslinking protein from Arabidopsis thaliana. Plant $\mathrm{J}$, 2000, 24: 625-636

37 Huang S, Robinson RC, Gao LY, Matsumoto T, Brunet A, Blanchoin L, Staiger CJ. Arabidopsis VILLIN1 generates actin filament cables that are resistant to depolymerization. Plant Cell, 2005, 17: 486-501

38 Spudich JA, Watt S. The regulation of rabbit skeletal muscle contraction. I. Biochemical studies of the tropomyosin-troponin complex with actin and the proteolytic fragments of myosin. J Biol Chem, 1971, 246: 4866-4871

39 Sluchanko NN, Gusev NB. 14-3-3 proteins and regulation of cytoskeleton. Biochemistry (Mosc), 2011, 75: 1528-1546

40 Wen F, Wang J, Xing D. A protein phosphatase 2A catalytic subunit modulates blue light-induced chloroplast avoidance movements through regulating actin cytoskeleton in Arabidopsis. Plant Cell Physiol, 2012, 53: 1366-1379

41 Ketelaar T, Allwood EG, Hussey PJ. Actin organization and root hair development are disrupted by ethanol-induced overexpression of $\mathrm{Ar}$ abidopsis actin interacting protein 1 (AIP1). New Phytol, 2007, 174: 57-62

42 Augustine RC, Pattavina KA, Tuzel E, Vidali L, Bezanilla M. Actin interacting protein 1 and actin depolymerizing factor drive rapid actin dynamics in Physcomitrella patens. Plant Cell, 2011, 23: 3696-3710

43 Shi M, Xie Y, Zheng Y, Wang J, Su Y, Yang Q, Huang S. Oryza sativa actin-interacting protein 1 is required for rice growth by promoting actin turnover. Plant J, 2013, 73: 747-760

44 Bernard O. Lim kinases, regulators of actin dynamics. Int J Biochem Cell Biol, 2007, 39: 1071-1076

Open Access This article is distributed under the terms of the Creative Commons Attribution License which permits any use, distribution, and reproduction in any medium, provided the original author(s) and source are credited.

\section{Supporting Information}

Figure S1 14-3-3 $\lambda$ does not bind F-actin and has no effect on actin polymerizing.

Figure S2 14-3-3 $\lambda$ does not directly affect actin depolymerizing activity of ADF1 in vitro.

Figure S3 RT-PCR analysis showed the expression level of $A D F 1$ in Col-0, adf1 and $A D F 1$ overexpression lines in 14-3-3 $\lambda$ mutant.

The supporting information is available online at life.scichina.com and link.springer.com. The supporting materials are published as submitted, without typesetting or editing. The responsibility for scientific accuracy and content remains entirely with the authors. 